\title{
DEMOGRAPHIC PREDICTION TOWARDS LEADERSHIP AND TEACHERS' COMMITMENT AND MOTIVATION IN THE EDUCATIONAL SECTOR
}

Afshan Jabeen $^{1 *}$, Muhammad Safdar Luqman', Syed Zia-ul-Islam³, Muhammad Asghar Khan ${ }^{4}$, Mehhwish Manzoor $^{5}$

${ }^{1 *}$ Senior Subject Specialist, School Education Department Punjab, Pakistan; ${ }^{2}$ Senior Instructor Physical Education, Elementary and Secondary Education, KP, Pakistan; ${ }^{3}$ Associate Professor, Department of Sports Sciences and Physical Education, University of Haripur, Pakistan; ${ }^{4}$ Instructor Physical Education, Elementary and Secondary Education, KP, Pakistan; ${ }^{5}$ Ph.D., Scholar, Department of Physical Education, University of Sindh, Jamshoro, Pakistan. Email: ${ }^{*}$ ajmalik54@gmail.com, ${ }^{2}$ safdarkhan821@ gmail.com, ${ }^{3}$ ziaulislamgr8@ gmail.com,

asgharkhan.bannu@gmail.com, ${ }^{5}$ mehwishmanzoor233@yahoo.com

Article History: Received on $30^{\text {th }}$ May 2021, Revised on $6^{\text {th }}$ June 2021, Published on $7^{\text {th }}$ June 2021

\begin{abstract}
Purpose of the study: The existing research study aimed to investigate the predictive effect of demographic traits, leadership styles on teachers' job commitment and motivation at the college level, in Punjab, Pakistan.

Methodology: The population of the study consisted of 576 male and female lecturers (PE). The total number of lecturers (PE) working in all Government colleges is 576, male lecturer (PE) is 366, and female lecturers (PE) are 210. Three self cum closed-ended questionnaires were designed for the data collection. Mean, standard deviation, Independent Samples Test, and One-way ANOVA were applied to test the formulated hypotheses.

Main Findings: It was found that there no positive impact of leadership styles of the head of institutions upon professional commitment and motivation of the lecturers based on demographics. It was also found that the study respondents regarding demographic attributes have significant group mean differences in opinion about the research variables under study.

Implications of the study: It was recommended that the most motivated and committed worker is the one who is senior, married, educated, and female, has a sound salary package, has served the institution for a long time- period and has a chance of professional development. It was also found that s/he performs his/her duty under an HOI who has more of a transformational leadership style than the rest and should be more transformational. Therefore HOI of the institutions should adopt a transformational leadership style to get maximum output from the employees.
\end{abstract}

Keywords: Demographics Prediction, Leadership Style, Job Commitment, Motivation.

\section{INTRODUCTION}

The current study aimed to examine the relationship among leadership styles, job commitment, motivation, and teachers' attributes. The current study concentrates on investigating the effect of transactional and transformational leadership on job performance concerning demographic traits in the educational education sector in Punjab, Pakistan. Job commitment and motivation are the vital ingredients of total job performance and play a vital role in achieving organizational goals and building a good repute of an institute. However, the experimental study reported regarding the above mention research variable but still, there is a need to investigate these phenomena especially in the field of education. Research Studies by Emery and Barker (2007) focused on the situational aspects that could affect the leader's behaviour. The subordinate behavior, demographics traits, task complexity, project size, organizational climate, and norms are some basic situational factors found to significantly affect a leader's behavior. Jena, Bhattacharyya, and Pradhan (2017) described that many researchers had focused mainly on transactional and transformational leadership for the last two decades. As the views to Bums, Transactional leadership occurs when approaching the leader's adherents to operate one thing for another thing. Such types of exchanges involve the association between the leaders and subordinates. Whereas, Senthamil and Palanichamy (2011) investigated that the transformational leader monitors and abuses the existing need of a potential believer.

In addition, although leadership is thought to a vital part of the requirement of higher quality delivery levels, the question arises of how leaders lead the educational organization which still uninvestigated with respect to the Pakistani context, leadership style has been discussed to include both main types, namely transformational and transactional leadership (iSolà, et al., 2016). Leadership is the main source of changing the abilities, qualities, and vision of group members with the personal abilities and resources of a leader. Kouzesand Posner (2011) described that in order to achieve the good common targets by inspiring and mobilizing others to perform some action is leadership in reality. So for helping, encouraging, and motivating teachers and learners to work enthusiastically towards educational institutions and their objectives is a process of leadership actually. Some research studies found that appropriate leadership and practices might be as inviting teachers' loyalty, commitment, and motivation.

There is a basic need to recognize the relationship between the education system and teachers in terms of the theoretical framework of leadership and its demographic attributes. The relevant literature to the education system indicates several 
variables that verified the administration leadership. Some traits of demographics such as gender, locality, teaching experience, marital status, and designation are taken as demographic variables in the present research. Likewise, if the HOIs are well contented demographically and build up leadership high level with their education system subsequently they will more likely to be dedicated, loyal to their profession (Rast \& Tourani, 2012).

\section{OBJECTIVES OF THE STUDY}

To examine the group mean differences in responses of respondents concerning the research variables.

\section{LITERATURE REVIEW}

Leadership plays an imperative role in motivating, inspiring, and empowering teachers as well as achieving organizational success. The person who commands or leads the organization or group as well as achieves the targeted goal by undertaking summative action is called a leader (Sun, Chen, \& Zhang, 2017). To establish a successful educational system, leadership contributes a lot. Any institution turns its direction in a positive way and institution, the educational staff is being motivated, inspired, and provide better role models. In this aspect of leadership, leadership is very effective in motivating teachers during individuals and shared learning. These learning types are an effective aspect of the educational institute's achievements (Leithwood \& Jantzi, 2006). Along with these, institutional leadership makes or breaks the motivation, commitment, and positivity of the teachers and students. So, professional commitment and motivation in any field are subjected to a peaceful and friendly environment. According to Jena, Bhattacharyya, and Pradhan (2017), commitment is the feeling of loyalty and passion of an employee towards its institute. It identifies the teacher's passion, devotion, and desire for hard work facilitate the idealization of their college.

\section{DEMOGRAPHIC FACTORS}

The review of literature of demographic variables (locality, gender, marital status, designation, teaching experience) have been reported a stable relationship with teachers' commitment and found that these affect teachers' commitment and motivation. Demographic traits have been selected due to their direct influence on teachers' commitment and motivation. Several studies in which demographic variables have been used to determine job commitment and motivation have indicated that they are strong predictors of job commitment and motivation (Malik, Javed, \& Hassan, 2017; Salami, 2008). Several research studies revealed that the impact of demographic variables was secondary to teachers' commitment and motivation. But, in few research studies indicated that demographic traits including marital status, locality, gender, teaching experience, educational and professional qualifications play a vital role in developing the leadership and staff' commitment and motivation (Omar, Self, Cole, Rashid, \& Puad, 2018; Zakeer, Munnawar \& Irfan, 2014)

\section{Gender}

A variety of variables of demographics have been included in different research studies. A research study by Akar (2018), Luqman, Rehman, Islam, and Khan, 2020), Feather and Rauter (2004) shown that female teachers had a higher level of organizational commitment than male teachers; yet, the rate of the difference was not at large. On the other side, some research studies by Ensher, Grant-Vallone, and Donaldson (2001) found that there is no difference between levels of organizational commitment of women and men. Benson and Griffin (1988) also found that female teachers have substantially less militant behaviors and attitudes than males.

\section{Locality}

The study also shows a significant relationship between professional commitment and location where teachers perform their assigned tasks/jobs. Teachers working in urban districts constantly shown less militant behaviors and attitudes as compare to the rural districts? Yet, another research study by Fiaz and Saqib (2017) found that there is no primary difference in the militant behaviours of teachers in urban and rural districts.

\section{Marital Status}

About the marital status of the employee, DiPrete and Buchmann (2013) in their research indicated that for unmarried workers without kids, $58 \%$ of males and $53 \%$ of females described that they intended to stay in uniform and more committed to their jobs. It indicates that married officers have more intention to leave their job due to their family attachment and circumstances than unmarried officers.

\section{Length of service}

A research study by Nugroho and Haryanto, (2019) showed a significant relationship between institutional commitment and the length of tenure of an individual in an institution. Another research by Canrinus et al. (2012) found that years of experience have a positive correlation with the professional commitment and motivation of teachers. In contrast, scholar Sezgin (2009) found that years of experience negatively related to the institutional commitment of teachers.

\section{Professional qualification}

$\underline{\text { Tillman and Tillman (2008) }}$ conducted a research study on the organizational commitment of elementary and secondary 
teachers and found that secondary teachers had lower levels of professional commitment than elementary teachers. On the other side, it was also found that there are no main differences between the militant levels of behaviours levels of the secondary and elementary teachers. However, Özdem (2012) found a positive relationship between militancy with the grade/scale level of teaching, for example, top-level of hostility in higher scales/grades).

\section{Educational qualification}

Many research studies have recommended that there is an inverse relationship between education level and organizational commitment, which means if an individual obtained a higher level of education, then he/she would be less committed to his/her organization, and He/she would be more committed to their profession or his/her union (Moses, Berry, Saab, \& Admiraal, 2017). A research study by Naseem, Nawaz, Khan, Khan, and Khan (2013) shows an indirect significant effect of education on the professional commitment of workers by enhancing work incentives but has a direct negative impact when work incentives or remuneration are held constantly. Finally, the author concluded that the present research outcomes in this field have not been consistent. The commitment and motivation were included in the current study because there are mainly homogeneous teaching levels among the lecturers, for example, four years of universitylevel education with two/three years of training or six years of education at the university level with two years of training.

Moreover, some lecturers have M.Phil or Ph.D. degree holders. They consider that their job is a mismatch with their academic qualification, so they are less committed and motivated with their profession and always seek another best option for them. This diversity of outcomes concerning the effects that location, gender, years of teaching experience, and grade/scale levels have on commitment and motivation of lecturers (PE) included interest level to the practical worth of adding these variables in the current study. Social status, family background, wife belonging demographic incorporated for logical and practical reasons were such as part-time/full-time job, enrolment in college, and experience as a professor union representative of all college-level teachers, which represent all the types of issues regarding teaching and promotion matters.

\section{HYPOTHESES OF THE STUDY}

1. The respondents about the gender have significant group mean differences in their opinion about the research variables under study $\left(\mathrm{H}_{1}\right)$.

2. The respondents about the marital status have significant group mean differences in their opinion about the research variables under study $\left(\mathrm{H}_{2}\right)$.

3. The respondents about the residence have significant group mean differences in opinion about the research variables under study $\left(\mathrm{H}_{3}\right)$.

4. The respondents regarding general qualifications have significant group mean differences in opinion about the research variables under study $\left(\mathrm{H}_{4}\right)$.

5. The respondents about the professional qualifications have significant group mean differences in opinion about the research variables under study $\left(\mathrm{H}_{5}\right)$.

6. The respondents about the experience have significant group mean differences in opinion about the research variables under study $\left(\mathrm{H}_{6}\right)$.

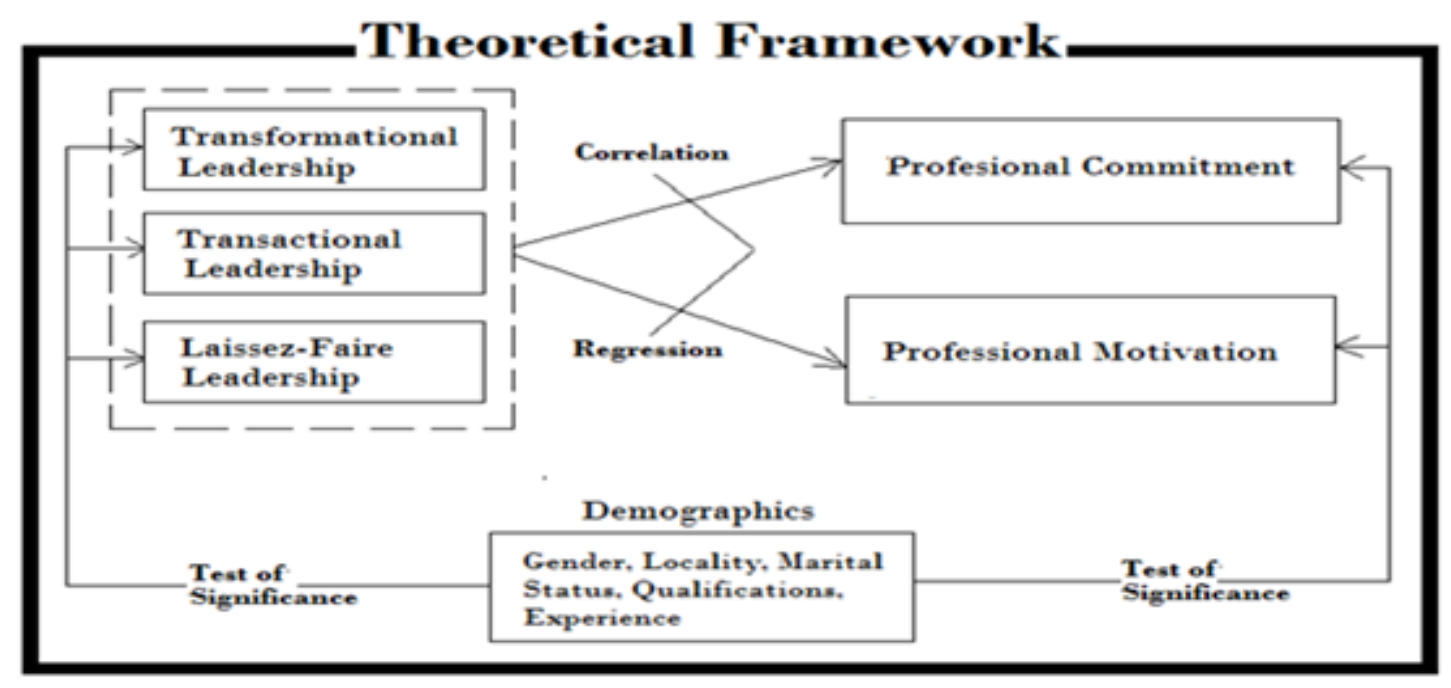

Figure 1: Theoretical framework 


\section{METHOD AND MATERIAL}

The current study was supported by the descriptive research design. Descriptive research design is the best form of research in which the nature of conditions and degree of the research study is described in detail. The population of this particular study was comprised of the teachers (PE) of all the Government colleges for boys and girls in Punjab province, Pakistan. There were 757 Government colleges for boys and girls situated in 36 districts of the Punjab province of Pakistan. The total number of teachers (PE) working in all Government colleges is 576, male lecturers (PE) are 366, and female lecturers (PE) are 210. The remaining 181 Govt. colleges had vacant posts due to no availability of LPEs. As the population was very small and easily approachable, so census method was taken as a sample of the study for data collection. As the present study dealt with three different variables like leadership styles, professional commitment, and professional motivation, the researcher developed and used three different self-made, close-ended on 5-point Likert scale for the data collection in the light of related literature and under the guidance of the supervisor. For the content validity, the initial versions of the scales were sent to the six experts of the department of social sciences of different public universities, Pakistan. The experts were requested to focus upon the validity and appropriateness of subfactors and the statements of respective scales. In light of the accurate observations and the positive recommendations of the doctors and experts, some of the ideas were deleted, edited, and modified with the consultation of the research supervisor accordingly. The researcher collected the questionnaires back through Email, Messenger, by hand, WhatsApp, and other postal services from all the participants after filling the scales. The researcher obtained the overall, $80 \%$ (439 out of 546) return response. In which 92(16.8\%) participants respond through what app, 57 (10.43\%) replied by Email, $125(22.9 \%)$ returned through the mail, 25 (4.6\%) respond through messenger, while by hand returned rate was recorded as $140(25.6 \%)$. The rate of response was representative. The collected data was put into the SPSS for analysis. Mean and standard deviation were used as descriptive statistics and T-Test, correlation, and One-way ANOVA were applied as inferential statistics to analyze the collected responses. Explanations are as follows:

\section{ANALYSES OF THE DATA}

$\mathbf{H}_{1}$ : The respondents with about the gender have significant group mean differences.

Table 1: Group Statistics (Gender)

\begin{tabular}{llllll}
\hline & Gender & $\mathbf{N}$ & Mean & Std. D & Std. E \\
\hline Transformational Leadership Style & Male & 292 & 3.62 & .696 & .041 \\
\cline { 2 - 6 } & Female & 147 & 4.18 & .500 & .041 \\
\hline Transactional Leadership Style & Male & 292 & 4.20 & .638 & .037 \\
\cline { 2 - 6 } & Female & 147 & 4.31 & .642 & .053 \\
\hline Laissez-faire Leadership Style & Male & 292 & 4.05 & .697 & .041 \\
\cline { 2 - 6 } & Female & 147 & 4.25 & .667 & .055 \\
\hline Professional Motivation & Male & 292 & 3.86 & .741 & .043 \\
\cline { 2 - 6 } & Female & 147 & 4.25 & .559 & .046 \\
\hline Professional Commitment & Male & 292 & 3.70 & .747 & .044 \\
\cline { 2 - 6 } & Female & 147 & 4.11 & .578 & .048 \\
\hline
\end{tabular}

Table 1(a): Independent Samples Test (Gender)

\begin{tabular}{lllllll}
\hline & & F & Sig. & T & df & Sig. \\
\hline Transformational Leadership Style & EVA & 17.288 & .000 & -8.602 & 437 & .000 \\
\cline { 2 - 7 } & EVNA & & & -9.568 & 385.982 & .000 \\
\hline Transactional Leadership Style & EVA & .752 & .386 & -1.616 & 437 & .107 \\
\cline { 2 - 7 } & EVNA & & & -1.613 & 291.001 & .108 \\
\hline Laissez-faire Leadership Style & EVA & .401 & .527 & -2.907 & 437 & .004 \\
\cline { 2 - 7 } & EVNA & & & -2.950 & 304.501 & .003 \\
\hline Professional Motivation & EVA & 11.685 & .001 & -5.691 & 437 & .000 \\
\cline { 2 - 7 } & EVNA & & & -6.234 & 372.348 & .000 \\
\hline Professional Commitment & EVA & 8.405 & .004 & -5.783 & 437 & .000 \\
\cline { 2 - 7 } & EVNA & & & -6.284 & 365.072 & .000 \\
\hline
\end{tabular}

The seventh hypothesis was about the gender-based group mean differences concerning research variables (transformational, transactional, and laissez-faire leadership, professional commitment, and professional motivation). In social sciences, demographic variables are commonly used for examining the variation in responses of respondents concerning the research variables under study. The results show that respondents about gender have significant group mean differences on almost all the research variables except transactional leadership styles. It means that males and females have differences in opinions concerning the research variables under consideration except for transactional leadership style. Therefore, from the results, the hypothesis H1 is partially accepted and substantiated. 


\section{Marital Status-based Differences of Opinion}

$\mathbf{H}_{2}$ : The respondents regarding marital status have significant group mean differences.

Table 2: Group Statistics (Marital Status)

\begin{tabular}{llllll}
\hline & Marital Status & N & Mean & SD & SEM \\
\hline Transformational Leadership Style & Married & 323 & 3.71 & .730 & .041 \\
\cline { 2 - 6 } & Single & 116 & 4.10 & .445 & .041 \\
\hline Transactional Leadership Style & Married & 323 & 4.21 & .638 & .036 \\
\cline { 2 - 5 } & Single & 116 & 4.33 & .640 & .059 \\
\hline \multirow{2}{*}{$\begin{array}{l}\text { Laissez-faire } \\
\text { Leadership Style }\end{array}$} & Married & 323 & 4.05 & .701 & .039 \\
\hline Professional Motivation & Single & 116 & 4.31 & .632 & .059 \\
\hline Professional Commitment & Married & 323 & 3.93 & .753 & .042 \\
\cline { 2 - 6 } & Single & 116 & 4.16 & .536 & .050 \\
\hline & Married & 323 & 3.75 & .754 & .042 \\
\cline { 2 - 5 } & Single & 116 & 4.08 & .550 & .051 \\
\hline
\end{tabular}

Table 2(a): Independent Samples Test (Marital Status)

\begin{tabular}{lllllll}
\hline & & F & Sig. & T & Df & Sig. \\
\hline Transformational Leadership Style & EVA & 32.010 & .000 & -5.424 & 437 & .000 \\
\cline { 2 - 7 } & EVNA & & & -6.759 & 333.499 & .000 \\
\hline Transactional Leadership Style & EVA & 1.596 & .207 & -1.712 & 437 & .088 \\
\cline { 2 - 7 } & EVNA & & & -1.709 & 202.561 & .089 \\
\hline Laissez-faire & EVA & 1.506 & .220 & -3.625 & 437 & .000 \\
\cline { 2 - 7 } Leadership Style & EVNA & & & -3.808 & 223.712 & .000 \\
\hline Professional Motivation & EVA & 16.829 & .000 & -3.118 & 437 & .002 \\
\cline { 2 - 7 } & EVNA & & & -3.647 & 285.086 & .000 \\
\hline Professional Commitment & EVA & 11.275 & .001 & -4.264 & 437 & .000 \\
\cline { 2 - 6 } & EVNA & & & -4.932 & 277.571 & .000 \\
\hline
\end{tabular}

The eighth hypothesis was about marital status-based group mean differences concerning research variables (transformational, transactional\& laissez-faire leadership, professional commitment, and professional motivation). The marital status is also widely used as the demographic variables concerning the group mean differences regarding various research variables. The results show that respondents with regard to marital status have significant group mean differences on almost all research variables. It means that married and unmarried respondents have significant differences in views about research variables under consideration (transformational, transactional, laissez-faire leadership, professional commitment, and professional motivation). Therefore, from the results, the hypothesis H8 is partially accepted and substantiated.

\section{Residence-based Differences of Opinion}

$\mathbf{H}_{3}$ : The respondents regarding residence have significant group mean differences.

Table 3: Group Statistics (Residence)

\begin{tabular}{llllll}
\hline & Residence & $\mathbf{N}$ & Mean & Std. Deviation & SE Mean \\
\hline Transformational Leadership Style & Urban & 254 & 3.54 & .688 & .043 \\
\cline { 2 - 5 } & Rural & 185 & 4.18 & .492 & .036 \\
\hline Transactional Leadership Style & Urban & 254 & 4.18 & .623 & .039 \\
\cline { 2 - 5 } & Rural & 185 & 4.32 & .656 & .048 \\
\hline \multirow{2}{*}{$\begin{array}{l}\text { Laissez-faire } \\
\text { Leadership Style }\end{array}$} & Urban & 254 & 4.02 & .701 & .044 \\
\cline { 2 - 5 } Professional Motivation & Rural & 185 & 4.25 & .659 & .048 \\
\hline Professional Commitment & Urban & 254 & 3.83 & .757 & .048 \\
\cline { 2 - 5 } & Rural & 185 & 4.21 & .569 & .042 \\
\hline & Urban & 254 & 3.64 & .740 & .046 \\
\cline { 2 - 5 } & Rural & 185 & 4.11 & .591 & .043 \\
\hline
\end{tabular}

Table 3(a): Independent Samples Test (Residence)

\begin{tabular}{lllllll}
\hline & & F & Sig. & T & df & Sig. \\
\hline Transformational Leadership Style & EVA & 17.468 & .000 & -10.726 & 437 & .000 \\
\cline { 2 - 7 } & EVNA & & & -11.285 & 436.883 & .000 \\
\hline Transactional Leadership Style & EVA & .003 & .958 & -2.261 & 437 & .024 \\
\hline
\end{tabular}




\begin{tabular}{lllllll}
\hline & EVNA & & & -2.243 & 384.581 & .025 \\
\hline \multirow{2}{*}{$\begin{array}{l}\text { Laissez-faire } \\
\text { Leadership Style }\end{array}$} & EVA & .096 & .757 & -3.575 & 437 & .000 \\
\cline { 2 - 7 } & EVNA & & & -3.610 & 409.755 & .000 \\
\hline Professional Motivation & EVA & 16.536 & .000 & -5.846 & 437 & .000 \\
\cline { 2 - 7 } & EVNA & & & -6.108 & 436.560 & .000 \\
\hline Professional Commitment & EVA & 8.313 & .004 & -7.200 & 437 & .000 \\
\cline { 2 - 6 } & EVNA & & & -7.456 & 433.278 & .000 \\
\hline
\end{tabular}

The ninth hypothesis was about residence-based group mean differences concerning research variables (transformational, transactional, laissez-faire leadership, professional commitment, and professional motivation). The residence is widely used as the demographic variables concerning the group mean differences about various research variables. The results show that respondents about residence have significant group mean differences on all research variables. It means that local and non-local respondents have significant differences in views about research variables under study (transformational, transactional, laissez-faire leadership styles, professional commitment, and professional motivation). Consequently, from the results, hypothesis H3 is partially accepted and substantiated.

\section{General Qualification-based Differences of Opinion}

$\mathbf{H}_{4}$ : Respondents about general qualifications have group mean differences.

Table 4: Group Statistics (GQUA)

\begin{tabular}{llllll}
\hline & GQUA1 & N & Mean & Std. Deviation & SE Mean \\
\hline \multirow{2}{*}{ Transformational Leadership Style } & MA/MSc & 306 & 3.64 & .688 & .039 \\
\cline { 2 - 6 } & M.Phil/Ph.D. & 133 & 4.21 & .504 & .044 \\
\hline \multirow{2}{*}{ Transactional Leadership Style } & MA/MSc & 306 & 4.20 & .629 & .036 \\
\cline { 2 - 6 } & M.Phil/ Ph.D. & 133 & 4.33 & .660 & .057 \\
\hline \multirow{2}{*}{ Laissez-faire } & MA/MSc & 306 & 4.05 & .689 & .039 \\
\cline { 2 - 6 } Leadership Style & M.Phil/Ph.D. & 133 & 4.27 & .681 & .059 \\
\hline Professional Motivation & MA/MSc & 306 & 3.87 & .732 & .042 \\
\cline { 2 - 6 } & M.Phil/ Ph.D. & 133 & 4.27 & .564 & .049 \\
\hline Professional Commitment & MA/MSc & 306 & 3.71 & .736 & .042 \\
\cline { 2 - 5 } & M.Phil/Ph.D. & 133 & 4.13 & .587 & .051 \\
\hline
\end{tabular}

Table 4(a): Independent Samples Test (GQUA)

\begin{tabular}{lllllll}
\hline & F & Sig. & T & Df & Sig. & F \\
\hline Transformational Leadership Style & EVA & 12.948 & .000 & -8.558 & 437 & .000 \\
\cline { 2 - 7 } & EVNA & & & -9.643 & 336.489 & .000 \\
\hline Transactional Leadership Style & EVA & .231 & .631 & -1.920 & 437 & .055 \\
\cline { 2 - 7 } & EVNA & & & -1.883 & 240.279 & .061 \\
\hline Laissez-faire & EVA & .961 & .328 & -3.086 & 437 & .002 \\
\cline { 2 - 7 } Leadership Style & EVNA & & & -3.100 & 253.788 & .002 \\
\hline Professional Motivation & EVA & 9.854 & .002 & -5.669 & 437 & .000 \\
\cline { 2 - 7 } & EVNA & & & -6.271 & 321.360 & .000 \\
\hline Professional Commitment & EVA & 5.416 & .020 & -5.827 & 437 & .000 \\
\cline { 2 - 6 } & EVNA & & & -6.362 & 311.010 & .000 \\
\hline
\end{tabular}

The tenth hypothesis was about general qualifications-based group mean differences concerning research variables (transformational, transactional, laissez-faire leadership, professional commitment \& professional motivation). The results show that respondents concerning general qualifications have significant group mean differences on all the research variables except transactional leadership style. It means that the qualifications of respondents have significant differences in views about research variables under study (transformational, transactional, laissez-faire leadership styles, professional commitment \& professional motivation). Thus, hypothesis H4 is partially accepted.

\section{Professional Qualification-based Differences of Opinion}

$\mathbf{H}_{5}$ : Respondents about professional qualifications have group mean differences.

Table 5: ANOVA Statistics (PQUA)

\begin{tabular}{llllllll}
\hline & & Sum $^{2}$ & Df & Mean $^{2}$ & F & Sig. \\
\hline \multirow{2}{*}{ Transformational Leadership Style } & Between Groups & 36.019 & 2 & 18.009 & 45.758 & .000 \\
\cline { 2 - 7 } & Within Groups & 171.600 & 436 & .394 & & \\
\cline { 2 - 7 } & Total & 207.619 & 438 & & & \\
\hline Transactional Leadership Style & Between Groups & 2.837 & 2 & 1.419 & 3.499 & .031 \\
\hline
\end{tabular}




\begin{tabular}{lllllll}
\hline & Within Groups & 176.775 & 436 & .405 & & \\
\cline { 2 - 6 } & Total & 179.612 & 438 & & & \\
\hline \multirow{2}{*}{$\begin{array}{l}\text { Laissez-faire } \\
\text { Leadership Style }\end{array}$} & Between Groups & 3.468 & 2 & 1.734 & 3.653 & .027 \\
\cline { 2 - 6 } & Within Groups & 206.908 & 436 & .475 & & \\
\cline { 2 - 6 } & Total & 210.375 & 438 & & & \\
\hline Professional Commitment & Between Groups & 13.366 & 2 & 6.683 & 14.064 & .000 \\
\cline { 2 - 6 } & Within Groups & 207.174 & 436 & .475 & & \\
\cline { 2 - 6 } & Total & 220.540 & 438 & & & \\
\hline Professional Motivation & Between Groups & 20.129 & 2 & 10.065 & 21.200 & .000 \\
\cline { 2 - 6 } & Within Groups & 206.994 & 436 & .475 & & \\
\cline { 2 - 6 } & Total & 227.124 & 438 & & & \\
\hline
\end{tabular}

Table 5(a): Group Statistics (PQUA)

\begin{tabular}{|c|c|c|c|c|c|c|c|}
\hline & & $\mathbf{N}$ & Mean & SD & SE & $95 \% \mathrm{C}$ & fidence \\
\hline & & & & & & Lower & Upper \\
\hline Transformational Leadership Style & JDPE & 141 & 3.60 & .638 & .054 & 3.49 & 3.70 \\
\hline & B.Ed & 198 & 4.12 & .562 & .040 & 4.04 & 4.20 \\
\hline & M.Ed & 100 & 3.49 & .728 & .073 & 3.35 & 3.63 \\
\hline & Total & 439 & 3.81 & .688 & .033 & 3.75 & 3.88 \\
\hline Transactional Leadership Style & JDPE & 141 & 4.12 & .632 & .053 & 4.02 & 4.23 \\
\hline & B.Ed & 198 & 4.29 & .662 & .047 & 4.20 & 4.39 \\
\hline & M.Ed & 100 & 4.30 & .590 & .059 & 4.18 & 4.41 \\
\hline & Total & 439 & 4.24 & .640 & .031 & 4.18 & 4.30 \\
\hline Laissez-faire & JDPE & 141 & 4.02 & .754 & .064 & 3.89 & 4.15 \\
\hline Leadership Style & B.Ed & 198 & 4.21 & .700 & .050 & 4.12 & 4.31 \\
\hline & M.Ed & 100 & 4.06 & .557 & .056 & 3.95 & 4.17 \\
\hline & Total & 439 & 4.12 & .693 & .033 & 4.05 & 4.18 \\
\hline Professional Commitment & JDPE & 141 & 3.80 & .716 & .060 & 3.68 & 3.92 \\
\hline & B.Ed & 198 & 4.18 & .588 & .042 & 4.10 & 4.26 \\
\hline & M.Ed & 100 & 3.87 & .824 & .082 & 3.71 & 4.04 \\
\hline & Total & 439 & 3.99 & .710 & .034 & 3.92 & 4.06 \\
\hline Professional Motivation & JDPE & 141 & 3.64 & .676 & .057 & 3.52 & 3.75 \\
\hline & B.Ed & 198 & 4.07 & .600 & .043 & 3.99 & 4.16 \\
\hline & M.Ed & 100 & 3.65 & .854 & .085 & 3.48 & 3.82 \\
\hline & Total & 439 & 3.84 & .720 & .034 & 3.77 & 3.91 \\
\hline
\end{tabular}

The eleventh hypothesis was about professional qualifications group mean differences concerning research variables (transformational, transactional, and laissez-faire leadership, professional commitment \& professional motivation). The results show that respondents with regard to professional qualifications have significant group mean differences on all the research variables. It means that the professional qualifications of the respondents have significant differences in views about research variables under study (transformational, transactional, and laissez-faire leadership styles, professional commitment \& professional motivation). Thus, hypothesis H5 is partially accepted.

\section{Experience-based Differences of Opinion}

$\mathbf{H}_{\mathbf{6}}$ : Respondents about experience have significant group mean differences.

Table 6: Group Statistics (Experience)

\begin{tabular}{llllllll}
\hline & & $\mathbf{N}$ & Mean & SD & SE & \multicolumn{2}{c}{ 95\% Confidence } \\
\hline \multirow{2}{*}{ Transformational Leadership Style } & & & & & & Lower & Upper \\
\cline { 2 - 7 } & $1-5$ & 145 & 3.61 & .638 & .053 & 3.50 & 3.71 \\
\cline { 2 - 7 } & $6-10$ & 111 & 4.10 & .536 & .051 & 4.00 & 4.20 \\
\cline { 2 - 7 } & $11 \&$ Above & 183 & 3.80 & .748 & .055 & 3.69 & 3.91 \\
\cline { 2 - 7 } & Total & 439 & 3.81 & .688 & .033 & 3.75 & 3.88 \\
\hline Transactional Leadership Style & $1-5$ & 145 & 4.13 & .630 & .052 & 4.02 & 4.23 \\
\cline { 2 - 7 } & $6-10$ & 111 & 4.28 & .681 & .065 & 4.15 & 4.41 \\
\cline { 2 - 7 } & $11 \&$ Above & 183 & 4.30 & .613 & .045 & 4.22 & 4.39 \\
\cline { 2 - 7 } & Total & 439 & 4.24 & .640 & .031 & 4.18 & 4.30 \\
\hline Laissez-faire Leadership Style & $1-5$ & 145 & 4.03 & .752 & .062 & 3.90 & 4.15 \\
\cline { 2 - 7 } & $6-10$ & 111 & 4.23 & .699 & .066 & 4.10 & 4.36 \\
\cline { 2 - 7 } & $11 \&$ Above & 183 & 4.12 & .631 & .047 & 4.03 & 4.22 \\
\hline
\end{tabular}




\begin{tabular}{llllllll}
\hline & Total & 439 & 4.12 & .693 & .033 & 4.05 & 4.18 \\
\hline Professional Commitment & $1-5$ & 145 & 3.82 & .713 & .059 & 3.70 & 3.94 \\
\cline { 2 - 7 } & $6-10$ & 111 & 4.16 & .569 & .054 & 4.05 & 4.26 \\
\cline { 2 - 7 } & 11 plus & 183 & 4.02 & .756 & .056 & 3.91 & 4.13 \\
\cline { 2 - 7 } & Total & 439 & 3.99 & .710 & .034 & 3.92 & 4.06 \\
\hline Professional Motivation & $1-5$ & 145 & 3.66 & .683 & .057 & 3.55 & 3.77 \\
\cline { 2 - 7 } & $6-10$ & 111 & 4.05 & .581 & .055 & 3.94 & 4.16 \\
\cline { 2 - 7 } & $11 \&$ Above & 183 & 3.85 & .789 & .058 & 3.74 & 3.97 \\
\cline { 2 - 7 } & Total & 439 & 3.84 & .720 & .034 & 3.77 & 3.91 \\
\hline
\end{tabular}

Table 6(a): ANOVA Statistics (Experience)

\begin{tabular}{lllllll}
\hline & & Sum $^{2}$ & df & Mean $^{\mathbf{2}}$ & F & Sig. \\
\hline Transformational Leadership Style & Between Groups & 15.470 & 2 & 7.735 & 17.551 & .000 \\
\cline { 2 - 6 } & Within Groups & 192.149 & 436 & .441 & & \\
\cline { 2 - 6 } & Total & 207.619 & 438 & & & \\
\hline \multirow{3}{*}{ Transactional Leadership Style } & Between Groups & 2.833 & 2 & 1.417 & 3.494 & .031 \\
\cline { 2 - 6 } & Within Groups & 176.779 & 436 & .405 & & \\
\cline { 2 - 6 } & Total & 179.612 & 438 & & & \\
\hline \multirow{3}{*}{ Laissez-faire Leadership Style } & Between Groups & 2.532 & 2 & 1.266 & 2.656 & .071 \\
\cline { 2 - 6 } & Within Groups & 207.843 & 436 & .477 & & \\
\cline { 2 - 6 } & Total & 210.375 & 438 & & & \\
\hline Professional Commitment & Between Groups & 7.544 & 2 & 3.772 & 7.721 & .001 \\
\cline { 2 - 6 } & Within Groups & 212.996 & 436 & .489 & & \\
\cline { 2 - 6 } & Total & 220.540 & 438 & & & \\
\hline Professional Motivation & Between Groups & 9.421 & 2 & 4.711 & 9.434 & .000 \\
\cline { 2 - 6 } & Within Groups & 217.702 & 436 & .499 & & \\
\cline { 2 - 6 } & Total & 227.124 & 438 & & \\
\hline
\end{tabular}

The twelfth hypothesis was about the teaching experience group mean differences about research variables (transformational, transactional, and laissez-faire leadership styles, professional commitment, and professional motivation). The results show that respondents about experience have significant group mean differences on all the research variables except transactional leadership style. It means that the professional qualifications of respondents have significant differences in views about research variables (transformational, laissez-faire leadership styles, professional commitment, and professional motivation). Thus, hypothesis H6 is partially accepted.

\section{DISCUSSION}

The particular research found that there are significant differences between the mean scores of male and female respondents regarding almost all research variables except transactional leadership style Furthermore, the findings of the study also show that the majority of the female gender was found to be more motivated and committed to their institution than the male LPEs. A research study by Fiaz and Saqib (2017); DiPrete and Buchmann (2013); Blankenship (2010) shown that female teachers had a higher level of organizational commitment than male teachers; yet, the rate of the difference was not at large. Another study by Özdem (2012) had also found the same results conducted on teachers. The study results also show that respondents with regard to marital status-based have significant group mean differences on almost all research variables. In addition to the analyzed data also found that unmarried LPEs are less committed than the married employees. A research study by Collie, Shapka, and Perry (2011) shown that there is a significant relationship between institutional commitment and the length of tenure of an individual in an institution. Another research by Naseem, Nawaz, Khan, Khan, and Khan (2013) conducted on teachers more specifically; also found that years of experience have a positive correlation with professional commitment and motivation of teachers. In addition to the analyzed data also found that unmarried LPEs are less committed than married employees. To describe this, one can say that married LPEs often have to support their families and have great responsibilities in life for which force them to be more motivated and committed to their institutions than the rest. Tebeian (2012) found that there is no main difference in the militant behaviors of teachers in unmarried LPEs and married. Similarly, the results also show that respondents about the residence-based have significant group mean differences on almost all research variables. Sezgin (2009) shown that there is a significant relationship between the professional commitment and location where teachers perform their assigned tasks/jobs. Teachers working in urban districts constantly shown less militant behaviors and attitudes than those teachers who were working in the rural districts? In addition to the same study, results show that respondents with regard to general qualifications-based have significant group mean differences on almost all research variables. It is also found that the LPEs who have low qualifications are more committed to their institutions than the rest. Many research studies have recommended that there is an inverse relationship between education level and organizational commitment (Keskes, 2014) 


\section{CONCLUSION OF THE STUDY}

The current study leads to investigate the association among leadership styles, job commitment, motivation, and teachers' personal attributes. The current study concentrates upon investigating the effect of transactional and transformational leadership on job performance with respect to demographic traits in the educational education sector in Punjab, Pakistan. ANOVA and One sample t-test revealed the significant groups mean differences between the dependent and the independent variables. Thus, leadership, employees' commitment, and motivation are strongly associated to different demographic traits of gender, marital status, locality, educational and professional qualification, and teaching experience. Furthermore, leadership styles have a number of adverse effects on the professional commitment and motivation of teaching faculty. The results of the present study, supported through the existing research studies revealed due to transformational leadership style, the positive variation found in the commitment and motivation. Similarly, the study concluded that there is a significant positive impact of the transactional leadership style on the motivation and commitment. The researcher concluded that there is a significant negative impact of laissez-faire leadership style on motivation and commitment of LPEs. However, the role of transformational leadership style in all the statistical procedure remained more significant due their artistic attributes. Furthermore, the investigator also concluded that teachers with higher education, having less teaching experience are less committed than others. Likewise, Married teachers are less motivated to their job than singles and rural teachers are more committed and motivated and having a specific attachment with their jobs as compare to urban teachers.

To sum up the study result it can be recommended that the most motivated and committed worker is the one who is senior, married, educated, female has sound salary package, has served the institution for a long time- period, has a chance of professional development and performs his/her duty under a HOI who has more of transformational leadership style than the rest and should be more transformational in nature. Therefore, there are certain recommendations as emerged from the conclusion of the study. This research recommends that professional commitment could be increased with appropriate leadership. It's also suggested that further researches should be done at various areas since this research is limited to higher education sector, Punjab province to verify whether these ingredients affecting teachers' job commitment and motivation would also be valid in those fields.

\section{ACKNOWLEDGEMENT}

Thank you to the higher education Commission and Gomal University Dera Ismail Khan of Pakistan who has provided research guidelines and funds to the research team. The research support and funding were given, including the category of centralization of basic research schemes in the first phase of the 2019 period.

\section{AUTHOR'S CONTRIBUTION}

Afshan Jabeen ${ }^{1}$, Muhammad Safdar Luqman ${ }^{2}$, Dr. Syed Zia-ul-Islam ${ }^{3}$, Muhammad Asghar Khan ${ }^{4}$, Mehhwish Manzoor ${ }^{5}$

Authors' Contribution: 1 - Study design; 2 - Data collection; 3- Statistical analysis; 4 - Manuscript preparation; 5Funds collection

\section{REFERENCES}

1. Akar, H. (2018). The relationships between quality of work life, school alienation, burnout, affective commitment and organizational citizenship: A study on teachers. European Journal of Educational Research, 7(2), 169-180. https://doi.org/10.12973/eu-jer.7.2.169

2. Blankenship, S. L. (2010). The consequences of transformational leadership and/or transactional leadership in relationship to job satisfaction and organizational commitment for active duty women serving in the Air Force Medical Service.

3. Canrinus, E. T., Helms-Lorenz, M., Beijaard, D., Buitink, J., \& Hofman, A. (2012). Self-efficacy, job satisfaction, motivation and commitment: Exploring the relationships between indicators of teachers' professional identity. European journal of psychology of education, 27(1),115-132. https://doi.org/10.1007/s10212-011-0069-2

4. Collie, R. J., Shapka, J. D., \& Perry, N. E. (2011). Predicting teacher commitment: The impact of school climate and social-emotional learning. Psychology in the Schools, 48(10), 1034-1048. https://doi.org/10.1002/pits.20611

5. DiPrete, T. A., \& Buchmann, C. (2013). The rise of women: The growing gender gap in education and what it means for American schools. Russell Sage Foundation.

6. Emery, C. R., \& Barker, K. J. (2007). The effect of transactional and transformational leadership styles on the organizational commitment and job satisfaction of customer contact personnel. Journal of organizational culture, communications and conflict, 11(1), 77.

7. Ensher, E. A., Grant-Vallone, E. J., \& Donaldson, S. I. (2001). Effects of perceived discrimination on job satisfaction, organizational commitment, organizational citizenship behavior, and grievances. Human resource development quarterly, 12(1), 53-72. $\quad$ https://doi.org/10.1002/15321096(200101/02)12:1<53::AID-HRDQ5>3.0.CO;2-G 
8. Feather, N. T., \& Rauter, K. A. (2004). Organizational citizenship behaviours in relation to job status, job insecurity, organizational commitment and identification, job satisfaction and work values. Journal of occupational and organizational psychology, 77(1), 81-94. https://doi.org/10.1348/096317904322915928

9. Fiaz, M., Su, Q., \& Saqib, A. (2017). Leadership styles and employees' motivation: Perspective from an emerging economy. The Journal of Developing Areas, 51(4), 143-156. https://doi.org/10.1353/jda.2017.0093

10. Jena, L. K., Bhattacharyya, P., \& Pradhan, S. (2017). Employee engagement and affective organizational commitment: Mediating role of employee voice among Indian service sector employees. Vision, 21(4), 356366. https://doi.org/10.1177/0972262917733170

11. Keskes, I. (2014). Relationship between leadership styles and dimensions of employee organizational commitment: A critical review and discussion of future directions. Intangible Capital, 10(1), 26-51. https://doi.org/10.3926/ic.476

12. Kouzes, J. M., \& Posner, B. Z. (2011). Credibility: How leaders gain and lose it, why people demand it (Vol. 203). John Wiley \& Sons. https://doi.org/10.1002/9781118983867

13. Leithwood, K. and Jantzi, D. (2006). Transformational School Leadership for Large-Scale Reform: Effects on students, teachers, and their classroom practices. School Effectiveness and School Improvement, 17 (2), 201-227. https://doi.org/10.1080/09243450600565829

14. Luqman, M. S., Rehman, J. U., Islam, Z. U., \& Khan, S. D. (2020). Effect of organizational climate upon the job performance of instructors' physical education. Pedagogy of Physical Culture and Sports, 24(2). https://doi.org/10.15561/26649837.2020.0204

15. Malik, W. U., Javed, M., \& Hassan, S. T. (2017). Influence of transformational leadership components on job satisfaction and organizational commitment. Pakistan Journal of Commerce and Social Sciences (PJCSS), 11(1), 147-166.

16. Moses, I., Berry, A., Saab, N., \& Admiraal, W. (2017). Who wants to become a teacher? Typology of studentteachers' commitment to teaching. Journal of Education for tEaching, 43(4), 444-457.

17. Naseem, B. Y., Nawaz, A., Khan, H., Khan, F., \& Khan, I. (2013). Determining the Demographic impacts on the Organizational Commitment of Academicians in the HEIs of DCs like Pakistan. European Journal of Sustainable Development, 2(2), 117-117. https://doi.org/10.14207/ejsd.2013.v2n2p117

18. Nugroho, A. P., \& Haryanto, A. T. (2019). The Role of Job Characteristics and Professional Commitment to Tenure and Performance (Study of Teachers in Public High Schools in Solo Raya). Journal of Indonesian Science Economic Research, 1(1), 26-31.

19. Omar, M. K., Self, M. J., Cole, K. L. M., Rashid, A. M., \& Puad, M. H. M. (2018). Job satisfaction and motivation to teach: Predicting intrinsic and extrinsic factors towards retaining career-switchers in the teaching profession. International Journal of Education, 3(16), 59-76.

20. Ozdem, G. (2012). The relationship between the organizational citizenship behaviors and the organizational and professional commitments of secondary school teachers. Journal of Global strategic management, 6(2), 47-64. https://doi.org/10.20460/JGSM.2012615773

21. Rast, S., \& Tourani, A. (2012). Evaluation of employees' job satisfaction and role of gender difference: An empirical study at airline industry in Iran. International Journal of Business and Social Science, 3(7).

22. Salami, S. O. (2008). Demographic and psychological factors predicting organizational commitment among industrial workers. The anthropologist, 10(1), 31-38. https://doi.org/10.1080/09720073.2008.11891026

23. Senthamil, R., \& Palanichamy, P. (2011). Transformational Leadership Styles and Employee Performance. Journal for Bloomers of Research, 3(2).

24. Sezgin, F. (2009). Relationships between teacher organizational commitment, psychological hardiness and some demographic variables in Turkish primary schools. Journal of Educational Administration, 47(5), 630-651. https://doi.org/10.1108/09578230910981099

25. Solà, G. J., i Badia, J. G., Hito, P. D., Osaba, M. A. C., \& García, J. L. D. V. (2016). Self-perception of leadership styles and behaviour in primary health care. BMC health services research, 16(1), 572. https://doi.org/10.1186/s12913-016-1819-2

Sun, J., Chen, X., \& Zhang, S. (2017). A review of research evidence on the antecedents of transformational leadership. Education Sciences, 7(1), 15. $\underline{\text { h https://doi.org/10.3390/educsci7010015 }}$

26. Tebeian, A. (2012). How to improve employee motivation and group performance through leadershipconceptual model. Annals of the University of Oradea, Economic Science Series, 21(1), 1092-1097.

27. Tillman, W. R., \& Tillman, C. J. (2008). And you thought it was the apple: A study of job satisfaction among teachers. Academy of educational leadership Journal, 12(3), 1.

28. Zakeer, A. K., Munnawar, N. K., Irfan, U. K. (2014). The impact of Leadership Styles on Innovation inthe Health Services. Public Policy and Administration Research, 4 (11), p- 78-84. 
Annexure 1 Questionnaire

Topic

\section{THE IMPACT OF LEADERSHIP STYLES OF HEAD OF INSTITUTIONS UPON PROFESSIONAL COMMITMENT AND MOTIVATION OF THE LECTURERS}

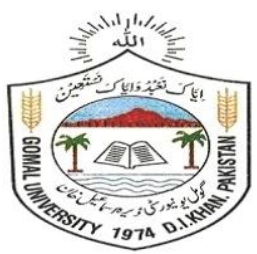

\section{Afshan Jabeen}

Department of Sports Sciences \& Physical Education

Gomal University, Dera Ismail Khan

\section{Dear Respondent!}

The questionnaire is used purely for Academic purposes thus your collaboration will help the student to fulfil the requirements for degree of $\mathrm{PhD}$ in Sports Sciences.

\section{Respondent Personal Profile}

1. Gender
3. Marital Status
5. Professional Qualification__

2. Residence

4.General Qualification

6. Experience

Note. How far do you Agree/Disagree with the following Statements using 5-Point Scale?

\begin{tabular}{lllll}
\hline Strongly Disagree & Disagree & Neutral & Agree & Strongly Agree \\
\hline 1 & 2 & 3 & 4 & 5 \\
\hline
\end{tabular}

\section{Transformational Leadership Style}

\begin{tabular}{lllllll}
\hline 1 & The principal acts in such manners that develop my respect for him/her. & 1 & 2 & 3 & 4 & 5 \\
\hline 2 & Principal always sets clear \& vivid teaching \& sport aims to be achieved. & 1 & 2 & 3 & 4 & 5 \\
\hline 3 & Principal renews my interest, trust \& optimism that is inspiring for me. & 1 & 2 & 3 & 4 & 5 \\
\hline 4 & Principal inspires me to follow new ideas relating to physical education. & 1 & 2 & 3 & 4 & 5 \\
\hline 5 & Principal guides and directs me when I requires the suitable directions. & 1 & 2 & 3 & 4 & 5 \\
\hline 6 & Principal provides knowledge and skills for accomplishing assignments. & 1 & 2 & 3 & 4 & 5 \\
\hline 7 & Principal assists me with respect to solving the problem regarding sports. & 1 & 2 & 3 & 4 & 5 \\
\hline 8 & Principal treats staff member as individual about his/her nature of job. & 1 & 2 & 3 & 4 & 5 \\
\hline 9 & Principal considers my personal interests \& abilities when assign tasks. & 1 & 2 & 3 & 4 & 5 \\
\hline 10 & Principal supports my attendance towards the professional development. & 1 & 2 & 3 & 4 & 5 \\
\hline
\end{tabular}

\section{Transactional Leadership Style}

\begin{tabular}{lllllll}
\hline 1 & The Principal monitors results whether objectives are attained or not? & 1 & 2 & 3 & 4 & 5 \\
\hline 2 & Principal observes and controls my performance to identify mistakes. & 1 & 2 & 3 & 4 & 5 \\
\hline 3 & The Principal takes necessary actions related to the sports development. & 1 & 2 & 3 & 4 & 5 \\
\hline 4 & The Principal takes positive measures before getting chronic situation. & 1 & 2 & 3 & 4 & 5 \\
\hline 5 & Principal shows satisfaction when I meet to set sports performance. & 1 & 2 & 3 & 4 & 5 \\
\hline 6 & Principal informs me potential contributions of my work to institution. & 1 & 2 & 3 & 4 & 5 \\
\hline 7 & Principal provides me rewards such as on achievement of performance. & 1 & 2 & 3 & 4 & 5 \\
\hline 8 & Principal imposes his/her powers when I cannot perform assigned work & 1 & 2 & 3 & 4 & 5 \\
\hline
\end{tabular}

\section{Laissez-faire Leadership Style}

\begin{tabular}{|c|c|c|c|c|c|c|}
\hline 1 & The Principal does not bother my activities if I do not make mistakes. & 1 & 2 & 3 & 4 & 5 \\
\hline 2 & The principal has never objected on my performance as Lecturer (PE). & 1 & 2 & 3 & 4 & 5 \\
\hline 3 & Principal does not constantly monitor my performance related to job. & 1 & 2 & 3 & 4 & 5 \\
\hline 4 & The Principal has complete trust in to deal with sports and class affairs. & 1 & 2 & 3 & 4 & 5 \\
\hline 5 & Principal let me make decision (sport, teaching) on my own interest. & 1 & 2 & 3 & 4 & 5 \\
\hline 6 & Principal gives me freedom to do what I want to do regarding sports. & 1 & 2 & 3 & 4 & 5 \\
\hline
\end{tabular}




\begin{tabular}{|c|c|c|c|c|c|c|}
\hline 7 & Principal feels will about taking initiative to conduct sports activities. & 1 & 2 & 3 & 4 & 5 \\
\hline 8 & Principal provides me timely feedback while performing my tasks. & 1 & 2 & 3 & 4 & 5 \\
\hline 9 & Principal gets himself involved in the instructional process of college. & 1 & 2 & 3 & 4 & 5 \\
\hline 10 & High interest of principal leads to high commitment with my work. & 1 & 2 & 3 & 4 & 5 \\
\hline
\end{tabular}

\section{Professional Commitment}

\begin{tabular}{lllllll}
\hline 1 & I have feelings of loyalty, passion and belongings for my institution. & 1 & 2 & 3 & 4 & 5 \\
\hline 2 & I am working with great zeal, devotion and dedication for institution. & 1 & 2 & 3 & 4 & 5 \\
\hline 3 & I have a good sense of belonging to my institute due to its care for me. & 1 & 2 & 3 & 4 & 5 \\
\hline 4 & I enthusiastically utilize my potentials for development of institution & 1 & 2 & 3 & 4 & 5 \\
\hline 5 & I have feelings of affection and love for my students in the institution. & 1 & 2 & 3 & 4 & 5 \\
\hline 6 & I fully concentrate on academic activities of my students and sports. & 1 & 2 & 3 & 4 & 5 \\
\hline 7 & I try to eliminate the academic deficiencies of my students in institution. & 1 & 2 & 3 & 4 & 5 \\
\hline 8 & I try to help students inside and outside the classroom in the institution. & 1 & 2 & 3 & 4 & 5 \\
\hline 9 & I have the emotional attachment and devotion with my profession. & 1 & 2 & 3 & 4 & 5 \\
\hline 10 & I always remain regular and pay full attention to my work in institution. & 1 & 2 & 3 & 4 & 5 \\
\hline
\end{tabular}

\section{Professional Motivation}

\begin{tabular}{lllllll}
\hline 1 & The teaching of physical education is an ideal job for me in institution. & 1 & 2 & 3 & 4 & 5 \\
\hline 2 & Teaching makes my life more meaningful within and outside institution. & 1 & 2 & 3 & 4 & 5 \\
\hline 3 & My job gives me freedom and power at the workplace in the institution. & 1 & 2 & 3 & 4 & 5 \\
\hline 4 & The institution where I work, provides me with the sufficient resources. & 1 & 2 & 3 & 4 & 5 \\
\hline 5 & My superiors do not try to block my advancement in the institution. & 1 & 2 & 3 & 4 & 5 \\
\hline 6 & I am happy with professional development prospects offered by HED. & 1 & 2 & 3 & 4 & 5 \\
\hline 7 & I avail opportunities of professional development provide by the HED. & 1 & 2 & 3 & 4 & 5 \\
\hline 8 & I have the good relationship with my heads/superiors and colleagues. & 1 & 2 & 3 & 4 & 5 \\
\hline 9 & I have a chance to maintain relations with all teachers in the institution. & 1 & 2 & 3 & 4 & 5 \\
\hline 10 & I have a respectable relationship with my students in the institution. & 1 & 2 & 3 & 4 & 5 \\
\hline
\end{tabular}

